\title{
Quantum field theory in curved spacetime, the operator product expansion, and dark energy
}

\author{
Stefan Hollands ${ }^{a *}$, Robert M. Wald ${ }^{{ }^{\dagger}}$ \\ ${ }^{a}$ School of Mathematics, Cardiff University, UK \\ ${ }^{b}$ Enrico Fermi Institute and Department of Physics \\ University of Chicago, Chicago, IL, USA
}

October 24, 2018

\begin{abstract}
To make sense of quantum field theory in an arbitrary (globally hyperbolic) curved spacetime, the theory must be formulated in a local and covariant manner in terms of locally measureable field observables. Since a generic curved spacetime does not possess symmetries or a unique notion of a vacuum state, the theory also must be formulated in a manner that does not require symmetries or a preferred notion of a "vacuum state" and "particles". We propose such a formulation of quantum field theory, wherein the operator product expansion (OPE) of the quantum fields is elevated to a fundamental status, and the quantum field theory is viewed as being defined by its OPE. Since the OPE coefficients may be better behaved than any quantities having to do with states, we suggest that it may be possible to perturbatively construct the OPE coefficients-and, thus, the quantum field theory. By contrast, ground/vacuum states-in spacetimes, such as Minkowski spacetime, where they may be defined-cannot vary analytically with the parameters of the theory. We argue that this implies that composite fields may acquire nonvanishing vacuum state expectation values due to nonperturbative effects. We speculate that this could account for the existence of a nonvanishing vacuum expectation value of the stress-energy tensor of a quantum field occurring at a scale much smaller than the natural scales of the theory.
\end{abstract}

*HollandsS@Cardiff.ac.uk

†rmwa@uchicago.edu 


\section{Quantum field theory in curved spacetime, the opera- tor product expansion, and dark energy}

Quantum field theory in curved spacetime is a theory wherein matter is treated fully in accord with the principles of quantum field theory, but gravity is treated classically in accord with general relativity. Despite its classical treatment of gravity, quantum field theory in curved spacetime has provided us with some of the deepest insights we presently have into the nature of quantum gravity. The main purpose of this essay is to argue that it also is providing us with significant insights into the nature of quantum field theory itself.

One of the key insights that has been obtained by the study of free (i.e., non-selfinteracting) quantum fields in curved spacetime is that - apart from stationary spacetimes or spacetimes with other very special properties - there is no unique, natural notion of a "vacuum state" or of "particles". Indeed, unless the spacetime is asymptotically stationary at early or late times, there will not, in general, even be an asymptotic notion of particle states. Consequently, it is essential that quantum field theory in curved spacetime be formulated in terms of the local field observables as opposed, e.g., to S-matrices. "Particle detectors" should be viewed as systems that interact with the local quantum fields [1], whose response can be calculated independently of any notion of "particles" that one may wish to introduce [2].

The usual formulations of quantum field theory in Minkowski spacetime (of both the rigorous and pragmantic kinds) rely on the following three key ingredients that do not generalize in an obvious way to curved spacetimes: (i) Poincare covariance of the quantum fields; (ii) positivity of total energy (spectrum condition); and (iii) the existence of a Poincare invariant state ("the vacuum"). With regard to (i), a generic curved spacetime will not possess any symmetries at all, so no spacetime symmetry requirements of any kind can be imposed on quantum fields in generic curved spacetimes. With regard to (ii), in the absence of a time translation symmetry, the total energy of even a classical field is highly timeslice dependent; since the energy density of a quantum field (in flat or curved spacetime) can be negative, and, in some simple examples involving free fields in curved spacetime, the integrated energy density is found to be negative, it seems clear that in curved spacetime no useful spectrum condition can be formulated in terms of the "total energy-momentum" of the quantum field. Finally, with regard to (iii), not only is "Poincare invariance" meaningless in curved spacetime, but one cannot expect that there exists a sensible criterion for picking out a special state of any kind in an arbitrary curved spacetime.

Nevertheless, in quantum field theory in curved spacetime, it is by now understood that there does exist a suitable replacement for the requirement, (i), of Poincare covariance of the quantum fields. Rather than require that the quantum fields be "specially covariant" in the sense of having the Poincare group act as a symmetry group, we require the quantum fields to be "generally covariant" in the sense that they be locally and covariantly constructed from the spacetime metric (as well as from other "background structure" such as space and time orientations and spin structure). In order to define this notion, it is essential that the quantum field theory be defined on all (globally hyperbolic) curved spacetimes, since in order to express the idea that the fields are locally determined by the spacetime metric, it is 
necessary to see how the theory changes when we change the metric in an arbitrary way. We then consider the following situation: Let $(M, g)$ and $\left(M^{\prime}, g^{\prime}\right)$ be two globally hyberbolic spacetimes that have the property that there exists an isometric imbedding, $\rho$, of $M$ into $M^{\prime}$, which also preserves causality relations and all of the other background structure. We require that there be a corresponding isomorphism, $\chi_{\rho}$, of the algebra of field observables on $(M, g)$ with the subalgebra of field observables on $\left(M^{\prime}, g^{\prime}\right)$ associated with the region $\rho[M]$. Furthermore, we require that $\chi_{\rho}$ maps each smeared quantum field $\phi^{(i)}(f)$ on $M$ to the corresponding quantum quantum field $\phi^{(i)}\left(\rho_{*}(f)\right)$ on $M^{\prime}$; see [3, 4, 5] for further discussion.

In addition, there exists a suitable generalization of the spectrum condition, (ii), to curved spacetime. The key idea is that the "positive energy" properties of quantum fields in Minkowski spacetime are directly related to their "positive frequency" properties, which, in turn, are directly related to the short-distance singularity structure of the of the $n$-point functions of the quantum fields. The positive frequency nature of the short-distance singularities of quantum fields can be characterized in a way that generalizes to curved spacetime. Thus, the spectrum condition used in Minkowski spacetime can be satisfactorily replaced by a microlocal spectrum condition in curved spacetime; see [6, 7, 8] for further discussion.

However, it is much less obvious how to find a suitable replacement for property (iii) in curved spacetime. In Minkowski spacetime, the existence of a unique, Poincare invariant state has very powerful consequences, so it is clear that a key portion of the content of quantum field theory in Minkowski spacetime would be missing if we failed to impose an analogous condition in curved spacetime. However, we do not believe that condition (iii) can be generalized to curved spacetime by a condition that postulates the existence of a preferred state with special properties.

Very recently, we have proposed [8] that the appropriate replacement of property (iii) for quantum field theory in curved spacetime is to postulate the existence of a suitable operator product expansion (OPE) of the quantum fields. By an OPE, we mean a family of formulae of the form

$$
\left\langle\phi^{\left(i_{1}\right)}\left(x_{1}\right) \cdots \phi^{\left(i_{n}\right)}\left(x_{n}\right)\right\rangle_{\omega} \approx \sum_{j} C_{(j)}^{\left(i_{1}\right) \ldots\left(i_{n}\right)}\left(x_{1}, \ldots, x_{n} ; y\right)\left\langle\phi^{(j)}(y)\right\rangle_{\omega} .
$$

Here, the $\phi^{(i)}$ denote the complete collection of fields in the theory (which may be of arbitrary tensorial or spinorial type), including all composite fields. The symbol \langle\rangle$_{\omega}$ denotes the expectation value in the state $\omega$. Each OPE coefficient $C_{(j)}^{\left(i_{1}\right) \cdots\left(i_{n}\right)}$ is a distribution on $M^{n+1}$ that is defined in some open neighborhood of the diagonal in $M^{n+1}$. The symbol " $\approx$ " in eq. (1) means that this equation holds in a suitably strong sense as an asymptotic relation in the limit that $x_{1}, \cdots, x_{n} \rightarrow y$. A precise definition of what is meant by this asymptotic relation is given in [8].

It was proposed in [8] that a quantum field theory should be viewed as being constructed from the list of quantum fields $\phi^{(i)}$ together with the family of all OPE coefficients

$$
\mathcal{C}(\mathbf{M}) \equiv\left\{C_{(j)}^{\left(i_{1}\right) \cdots\left(i_{n}\right)}\left(x_{1}, \ldots, x_{n} ; y\right)\right\},
$$


where $\mathbf{M}$ denotes all of the background structure, i.e., the spacetime $(M, g)$ together with choices of space and time orientations and spin structure. Given $C(\mathbf{M})$, the quantum field algebra of observables, $\mathcal{A}(\mathbf{M})$, is then constructed by starting with the free algebra Free $(\mathbf{M})$ generated by the smeared fields $\phi^{(i)}(f)$ and factoring it by certain relations. These relations consist of some "universal" relations that do not depend on the particular theory under consideration (such as linearity of $\phi^{(i)}(f)$ in $f$ ) together with certain relations that arise from the OPE. The precise construction of $\mathcal{A}(\mathbf{M})$ is given in [8]. The state space $\mathcal{S}(\mathbf{M})$ is then defined to be the subspace of the space of all linear, functionals $\omega: \mathcal{A}(\mathbf{M}) \rightarrow \mathbb{C}$ that are positive in the sense that $\omega\left(A^{*} A\right) \equiv\left\langle A^{*} A\right\rangle_{\omega} \geq 0$ for all $A \in \mathcal{A}(\mathbf{M})$, that satisfy a microlocal spectrum condition, and that satisfy the OPE relations, eq. (1).

The collection of OPE coefficients $\mathcal{C}(\mathbf{M})$ is, of course, not arbitrary but must satisfy certain general properties, which, in effect, become the "axioms" of quantum field theory in curved spacetime. The key properties that the OPE coefficients are required to satisfy include the following: (1) Each $C_{(j)}^{\left(i_{1}\right) \cdots\left(i_{n}\right)}\left(x_{1}, \ldots, x_{n} ; y\right)$ must be locally and covariantly constructed from the background structure M. (2) Each $C_{(j)}^{\left(i_{1}\right) \cdots\left(i_{n}\right)}\left(x_{1}, \ldots, x_{n} ; y\right)$ must satisfy a microlocal spectrum condition. (3) The coefficient, $C_{(\mathbf{1})}^{(i)\left(i^{\star}\right)}$, of the identity element, 1, must be the most singular OPE coefficient appearing on the right side of eq. (1) in the expansion of $\phi^{(i)}\left(x_{1}\right) \phi^{(i) *}\left(x_{2}\right)$. Furthermore, for $i \neq \mathbf{1}$, this coefficient must be singular in the sense of having positive scaling degree as $x_{1}, x_{2} \rightarrow y$. (4) If we let $x_{1}, \cdots, x_{n} \rightarrow y$ at different rates, then the $C_{(j)}^{\left(i_{1}\right) \cdots\left(i_{n}\right)}\left(x_{1}, \ldots, x_{n} ; y\right)$ must satisfy an "associativity condition" corresponding to what one would formally obtain by first performing an OPE for the subset of points that merge together the fastest, then performing an OPE on the resulting product of operators for the (merged) points that merge the second-fastest, etc. A precise statement of this condition and a complete enumeration of all of the other conditions we require for an OPE can be found in [8].

The type of operator product expansion that we require is known to hold in free field theory and to hold order by order in perturbation theory for interacting quantum fields in curved spacetime [9]. Thus, what we have proposed can be viewed as elevating the OPE to the status of a fundamental property of quantum fields. Although the assumption of the existence of an OPE in quantum field theory in curved spacetime is remarkably different in nature from the assumption of the existence of a Poincare invariant state in quantum field theory in Minkowski spacetime, the OPE plays a role similar to that of the existence of a Poincare invariant state in analyses and proofs; roughly speaking the coefficient of the identity, $C_{(\mathbf{1})}^{\left(i_{1}\right) \cdots\left(i_{n}\right)}\left(x_{1}, \ldots, x_{n} ; y\right)$, in the OPE in curved spacetime plays a role similar to that of the vacuum expectation value $\left\langle 0\left|\phi^{\left(i_{1}\right)}\left(x_{1}\right) \cdots \phi^{\left(i_{n}\right)}\left(x_{n}\right)\right| 0\right\rangle$ in Minkowski spacetime.

Using our new formulation of quantum field theory based on the existence of a suitable OPE, we have proven [8] curved spacetime versions of the spin-statistics theorem and the PCT theorem. Interestingly, the PCT theorem in curved spacetime has a significantly different character than the usual Minkowski version. The Minkowski version asserts the existence of an (anti-linear) symmetry associated with the PT isometry of Minkowski spacetime, which takes quantum fields into their charge conjugates. The curved spacetime version asserts the existence of a symmetry relating the quantum field theory defined 
on an arbitrary background structure $\mathbf{M}$ to the theory defined on the background structure $\mathbf{M}^{\prime}$ obtained from $\mathbf{M}$ by keeping the spacetime manifold, spacetime metric, and spacetime orientation the same, but reversing the time orientation. (This symmetry also maps fields to their charge conjugates.) Thus, for example, the curved spacetime version of the PCT theorem asserts that for every process that can occur in an expanding universe, there is a corresponding process (defined by the PCT symmetry) that occurs in the corresponding contracting universe (obtained by reversing the time orientation). We get a "same universe" version of the PCT theorem only in the case of a spacetime (such as Minkowski spacetime) that admits an isometry that preserves the spacetime orientation but reverses the time orientation; by combining the PCT symmetry (which takes $\mathcal{A}(\mathbf{M})$ to $\mathcal{A}\left(\mathbf{M}^{\prime}\right)$ ) with the symmetry arising from such a spacetime isometry (which takes $\mathcal{A}\left(\mathbf{M}^{\prime}\right)$ to $\mathcal{A}(\mathbf{M})$ ), we obtain a symmetry acting on $\mathcal{A}(\mathbf{M})$.

However, the potentially most significant ramifications of our new formulation of quantum field theory in curved spacetime concern the nature of quantum field theory itself. In our new formulation the existence of a "preferred state" no longer plays any role in the formulation of quantum field theory. States are inherently non-local in character, and the replacement of the existence of a preferred state by the existence of a suitable OPE along with the replacement of Poincare invariance by the condition that the quantum fields be local and covariant, and the replacement of the spectrum condition by the microlocal spectrum condition-yields a formulation of quantum field theory that is entirely local in nature. In this way, the formulation of quantum field theory becomes much more analogous to the formulation of classical field theory. Indeed, one can view a classical field theory as being specified by providing the list of fields $\phi^{(i)}$ occuring in the theory and the list of local, partial differential relations satisfied by these fields. Solutions to the classical field theory are then suitably regular sections of the appropriate vector bundles that satisfy the partial differential relations. Similarly, in our framework, a quantum field theory is specified by providing the list of fields $\phi^{(i)}$ occuring in the theory and the list of local, OPE relations satisfied by these fields. Thus, the OPE relations play a role completely analogous to the role of field equations in classical field theory. States-which are the analogs of solutions in classical field theory - are suitably regular (in the sense of satisfying the microlocal spectrum condition) positive linear maps on the field algebra that satisfy the OPE relations. It is worth noting that in classical field theory, the field equations always manifest all of the symmetries of the theory, even in cases where there are no solutions that manifest these symmetries. Similarly, in our formulation of quantum field theory, the OPE relations that define the theory should always respect the symmetries of the theory [10], even if no states happen to respect these symmetries.

Our viewpoint on quantum field theory is more restrictive than standard viewpoints in that we require the existence of an OPE. On the other hand, it is less restrictive in that we do not require the existence of a ground state. This latter point is best illustrated by considering a free Klein-Gordon field $\varphi$ in Minkowski spacetime

$$
\left(\square-m^{2}\right) \varphi=0,
$$

where the mass term, $\mathrm{m}^{2}$, is allowed to be positive, zero, or negative. In the standard 
viewpoint, a quantum field theory of the free Klein-Gordon field does not exist in any dimension when $m^{2}<0$ and does not exist in $D=2$ when $m^{2}=0$ on account of the nonexistence of a Poincare invariant state. However, there is no difficulty in specifying OPE relations that satisfy our axioms for all values of $m^{2}$ and all $D \geq 2$. In particular, for $D=4$ we can choose the OPE-coefficient $C$ of the identity in the OPE of $\varphi\left(x_{1}\right) \varphi\left(x_{2}\right)$ to be given by

$$
\begin{aligned}
& C\left(x_{1}, x_{2} ; y\right)= \\
& \frac{1}{4 \pi^{2}}\left(\frac{1}{\Delta x^{2}+i 0 t}+m^{2} j\left[m^{2} \Delta x^{2}\right] \log \left[\mu^{2}\left(\Delta x^{2}+i 0 t\right)\right]+m^{2} h\left[m^{2} \Delta x^{2}\right]\right),
\end{aligned}
$$

where $\Delta x^{2}=\left(x_{1}-x_{2}\right)^{2}$ and $t=x_{1}^{0}-x_{2}^{0}$. Here $\mu$ is an arbitrarily chosen mass scale and $j(z) \equiv \frac{1}{2 i \sqrt{z}} J_{1}(i \sqrt{z})$ is an analytic function of $z$, where $J_{1}$ denotes the Bessel function of order 1 . Furthermore, $h(z)$ is the analytic function defined by

$$
h(z)=-\pi \sum_{k=0}^{\infty}[\psi(k+1)+\psi(k+2)] \frac{(z / 4)^{k}}{k !(k+1) !} .
$$

with $\psi$ the psi-function. This formula for the OPE coefficient-as well as the corresponding formulas for all of the other OPE coefficients-is as well defined for negative $m^{2}$ as for positive $m^{2}$. Existence of states satisfying all of the OPE relations for negative $m^{2}$ can be proven by the deformation argument of [11], using the fact that such states exist for positive $m^{2}$.

The potential importance of the above example is that it explicitly demonstrates that the local OPE coefficients can have a much more regular behavior under variations of the parameters of the theory as compared with state-dependent quantities, such as vacuum expectation values. The OPE coefficients in the above example are analytic in $m^{2}$. On the other hand, the 2-point function of the global vacuum state is, of course, defined only for $m^{2} \geq 0$ and is given by

$$
\begin{aligned}
& \left\langle 0\left|\varphi\left(x_{1}\right) \varphi\left(x_{2}\right)\right| 0\right\rangle= \\
& \frac{1}{4 \pi^{2}}\left(\frac{1}{\Delta x^{2}+i 0 t}+m^{2} j\left[m^{2} \Delta x^{2}\right] \log \left[m^{2}\left(\Delta x^{2}+i 0 t\right)\right]+m^{2} h\left[m^{2} \Delta x^{2}\right]\right) .
\end{aligned}
$$

This behaves non-analytically in $m^{2}$ at $m^{2}=0$ on account of the $\log m^{2}$ term. In other words, in free Klein-Gordon theory, vacuum expectation values cannot be constructed perturbatively by expanding about $m^{2}=0$-as should be expected, since no vacuum state exists for $m^{2}<0$ - but there is no difficulty in perturbatively constructing the OPE coefficients by expanding about $m^{2}=0$.

In quantum field theory in Minkowski spacetime, attention is usually focused upon quantities that involve states, such as S-matrix elements. Since the states of interest cannot be expected to vary analytically with the parameters of the theory, perturbation expressions for quantities such as S-matrix elements cannot be expected to converge, and there is ample evidence that they do not. However, the above considerations suggest the possibility that the 
OPE coefficients may have much better behavior than quantities associated with states, and that the perturbation series for the OPE coefficients may converge. In other words, we are suggesting the possibility that within our framework, it may be possible to perturbatively construct interacting quantum field theories. Aside from the free Klein-Gordon example above, the only evidence we have in favor of convergence of perturbative expansions for OPE coefficients is the example of super-renormalizable theories, such as $\lambda \varphi^{4}$-theory in two spacetime dimensions [12]. Here, only finitely many terms in a perturbative expansion can contribute to any OPE coefficient up to any given scaling degree, so convergence (up to any given scaling degree) is trivial. By contrast, for $\lambda \varphi^{4}$-theory in two spacetime dimensions, the rigorously constructed, non-perturbative ground state $n$-point functions can be proven to be non-analytic at $\lambda=0$; see e.g. [13]. Of course, even if we had the complete list of OPE coefficients, we would still need to construct states, which cannot be done perturbatively. Nevertheless, it would be potentially very useful to have the OPE coefficients even if one did not have states (or even an existence proof for states) - just as in classical field theory it is useful to have the field equations even if one does not have a method for finding solutions.

In cases-such as free Klein-Gordon theory above-where the OPE coefficients can be chosen to be analytic in the parameters of the theory, it seems natural to require that the theory be defined so that this analytic dependence holds. This requirement has some potentially major ramifications, which we now discuss. Since a vacuum expectation value of a products of fields (i.e., a correlation function) would be expected to have a non-analytic dependence on the parameters of the theory, it follows that if the OPE coefficients have an analytic dependence on these parameters, then, even in Minkowski spacetime, some of the fields appearing on the right side of the OPE eq. (1) must acquire a nonvanishing vacuum expectation value, at least for some values of the parameters. This point is well illustrated by the above Klein-Gordon example. It is natural to identify the next term (i.e., the term beyond the identity term) in the OPE of $\varphi\left(x_{1}\right) \varphi\left(x_{2}\right)$ as being $\varphi^{2}$ (with unit coefficient), i.e.,

$$
\varphi\left(x_{1}\right) \varphi\left(x_{2}\right) \sim C\left(x_{1}, x_{2} ; y\right) \mathbf{1}+\varphi^{2}(y)+\ldots,
$$

with $C\left(x_{1}, x_{2} ; y\right)$ given by eq. (4). This corresponds to the usual "point-splitting" definition of $\varphi^{2}$, except that $C\left(x_{1}, x_{2} ; y\right)$ now replaces $\left\langle 0\left|\varphi\left(x_{1}\right) \varphi\left(x_{2}\right)\right| 0\right\rangle$. If we take the vacuum expectation value of this formula (for $m^{2} \geq 0$, when a vacuum state exists) and compare it with eq. (6), we obtain

$$
\left\langle 0\left|\varphi^{2}\right| 0\right\rangle=-\frac{m^{2}}{16 \pi^{2}} \log \left(m^{2} / \mu^{2}\right) .
$$

Thus, we cannot set $\left\langle 0\left|\varphi^{2}\right| 0\right\rangle=0$ for all values of $m^{2}$. A similar calculation for the stressenergy tensor of $\varphi$ yields

$$
\left\langle 0\left|T_{a b}\right| 0\right\rangle=\frac{m^{4}}{64 \pi^{2}} \log \left(m^{2} / \mu^{2}\right) \eta_{a b}
$$

\footnotetext{
${ }^{1}$ In order to do so, it will be necessary to define the basis fields $\phi^{(i)}$ appropriately and also to parametrize the theory appropriately (since a theory with an analytic dependence on a parameter could always be made to appear non-analytic by a non-analytic reparametrization).
} 
As in other approaches, the freedom to choose the arbitrary mass scale $\mu$ in eq. (4) gives rise to a freedom to choose the value of the "cosmological constant term" in eq. (9). However, unlike other approaches, there is no freedom to adjust the value of the cosmological constant when $m^{2}=0$ (i.e., we unambiguously obtain $\left\langle 0\left|T_{a b}\right| 0\right\rangle=0$ in Minkowski spacetime in that case), and the $m^{2}$-dependence of the cosmological constant is fixed (since $\mu$ is not allowed to depend upon $m^{2}$ ).

Although the example of the free Klein-Gordon field is, of course, too trivial to be realistic, it serves to illustrate the conflict between the expected non-analytic behavior of the left side of eq. (1) and the conjectured analytic behavior of the OPE coefficients-a conflict that can be resolved only if the operators appearing on the right side of eq. (1) generically acquire a nonvanishing vacuum expectation value. A much more interesting example arises for interacting field theories, such as non-abelian gauge theories, where "nonperturbative" effects are known to arise. If such nonperturbative effects contribute at finite scaling degree to the field correlation functions appearing on the left side of eq. (1), then it is natural to expect that they will similarly contribute to the vacuum expectation values of the fields appearing on the right side. In particular, they may contribute to the vacuum expectation value of the stress-energy tensor.

One of the great mysteries of modern cosmology is to account for the acceleration of the present universe. In order to explain the observed acceleration, one must postulate the existence of "dark energy", a component of matter that is distributed uniformly throughout the universe and has large negative pressure. There is growing evidence that "dark energy" corresponds to a cosmological constant term in Einstein's equation, i.e., a stress-energy tensor proportional to the metric. While it is not difficult to imagine how a "vacuum energy" contribution of this general form to the stress-energy tensor could arise, it is very difficult to imagine how one could account for the incredible mis-match of scales between the value of the cosmological constant required to explain the observed acceleration-corresponding to a length scale of order the Hubble radius - and the natural length scales occurring in particle physics. We are proposing that this mis-match might be explained if, as we have argued above, the "vacuum energy" is associated with non-perturbative effects, since nonperturbative effects can potentially be extremely small compared with the natural scales appearing in a theory. This possibility appears worthy of further investigation.

Acknowledgments: This research was supported in part by NSF Grant PHY04-56619 to the University of Chicago.

\section{References}

[1] W.G. Unruh, “Notes on Black Hole Evaporation,” Phys. Rev. D14, 870-892 (1976).

[2] W.G. Unruh and R. M. Wald, "What Happens When an Accelerating Observer Detects a Rindler Particle?" Phys. Rev. D29, 1047-1056 (1984). 
[3] S. Hollands and R. M. Wald: "Local Wick Polynomials and Time Ordered Products of Quantum Fields in Curved Space," Commun. Math. Phys. 223, 289-326 (2001), [gr-qc/0103074].

[4] S. Hollands and R. M. Wald, "Existence of local covariant time-ordered-products of quantum fields in curved spacetime," Commun. Math. Phys. 231, 309-345 (2002), [gr-qc/0111108].

[5] R. Brunetti, K. Fredenhagen and R. Verch, "The generally covariant locality principle: A new paradigm for local quantum physics," Commun. Math. Phys. 237, 31 (2003), [math-ph/0112041]; see also K. Fredenhagen, "Locally covariant quantum field theory," arXiv:hep-th/0403007].

[6] R. Brunetti, K. Fredenhagen and M. Köhler: "The microlocal spectrum condition and Wick polynomials on curved spacetimes," Commun. Math. Phys. 180, 633-652 (1996).

[7] R. Brunetti and K. Fredenhagen: "Microlocal Analysis and Interacting Quantum Field Theories: Renormalization on physical backgrounds," Commun. Math. Phys. 208, 623-661 (2000).

[8] S. Hollands and R. M. Wald, "Axiomatic Quantum Field Theory in Curved Spacetime," arXiv:0803.2003.

[9] S. Hollands, "The operator product expansion for perturbative quantum field theory in curved spacetime," Commun. Math. Phys. 273, 1 (2007) [arXiv:gr-qc/0605072].

[10] See appendix in: C. Bernard, A. Duncan, J. LoSecco, S. Weinberg: "Exact spectralfunction sum rules", Phys. Rev. D 12, 792 - 804 (1975).

[11] S.A. Fulling, F.J. Narcowich, and R.M. Wald: "Singularity Structure of the TwoPoint Function in Quantum Field Theory in Curved Spacetime, II," Ann. Phys. 136 243 (1981).

[12] S. Hollands and C. Kopper, in progress.

[13] V. Rivasseau, "From perturbative to constructive renormalization," Princeton, USA: Univ. Pr. (1991) 336 p. (Princeton series in physics). 